\title{
Study of Image Compression Based on Fast Lifting Waveletransform
}

\author{
Changzheng Liu \\ College of Computer Science and Technology \\ Harbin University of Science and Technology \\ Harbin, P.R.China \\ liuchangzhengy@163.com
}

\author{
Qian Ding \\ College of Management \\ Harbin University of Science and Technology \\ Harbin, P.R.China \\ 13351985596@163.com
}

\begin{abstract}
Image matching is an effective auxiliary location method for the precise guide weapon system. The image sensor of image matching system is used to real-time gather the object image around target or that throughout the flight way, which will be compared with the reference data of the upper computer in the missile, to process and locate the correct position of the target relative to the missile. The performance of the image matching system is vitally interrelated to the geographic and conditions (Ground image characteristics) of the operation region. So the basic operation condition of the image matching system and matching region selection in the special geographic conditions is regarded as the main contents to study. Taken the developing demands of an important national defense weapon system as the background, we carry out a series of theory analysis and simulation study base on the actual data, in order to improve the matching accuracy of the down-look image matching system and determine the main factors influencing the operation performance of the image matching system in further. And we build a set of functional relationship relative to the performance and specifications of the image matching system, image statistic parameters and operation conditions. By analyzing and verifying these functions relationship, finally we point out the parameters influencing the image matching system under different environmental conditions, and point out principle thought and method of selecting image matching area according to these parameters. These study results have been used and proved in the product development. And it provides the basis for us to determine the selection regulations of matching area of downlook system, laying a foundation for improving the operation performance of the image matching system in different environmental conditions.
\end{abstract}

Keywords-wavelet transform; fast lifting; intrusion detection; analysis of data-package

\section{INTRODUCTION}

The client/server structure has become the most popular system model, it seems particularly important as to how to develop secure and highly efficient database systems, providing strong and fast multi-user client/server applications, effectively ensuring the irritability of the system, protecting customers' investment to information systems. To deal with these controversies, three- tiered client/server model has emerged and is widely applied. Three- tiered (n- tiered) structure concept is becoming a hotspot in current database system applications research [1].

This article analyzed the basic concept system, go deep into the application of technology such as ActiveX, IDC and ASP in the three- tiered database, putting forward many ways of information system design, and subsequently using examples to detail the process of this model used in the information system development [2]. This article can be divided into three parts: the first is concept discussion, consists of Chapter One, introduction and Chapter Two, overview of three- tiered client/server model, focused on the background of the creation of client/server model, concept definition, essential elements, and managing process and implementation issues of the information systems [3]. The second part is technology, consists of Chapter Three and Chapter Four, mainly discussed and addressed the features, structures, ways of implementing model establishment and Web-based database appliance of three-tiered structure. The third part is application examples, including Chapter Five. The author completed the design of a three-tiered client/server model information system according to the task of the construction of scientific research information systems, network platform based on Microsoft Windows NT/2000 and TRS full text searching database system [4].

This assay is focuses on part of access network technology. The main contents are as the follows.

To discuss the function of the forthcoming copper loop, city cable channel's once and twice parameters are analyzed and calculated, and simple analytic model are used to describe the characteristics of channel. The transformation of parameter related to applied HDSL technology in higher band of frequency, and the influences of near reaction and twisted subscriber line are mainly analyzed [5].

\section{DISCRETE WAVELET TRANSFORM}

To discuss the influence of transmission environment, the noise of the present subscribe channel and its influence on digital signal transmission are analyzed. The possible influence of pulse noise on HDSL technology's application is analyzed quantitatively, subscribe channel's interference voice is analyzed, and the influence of bridged tap on 
subscriber loop when it is applied under the digital condition of subscriber channel is calculated.

On the basis of above-mentioned analysis of channel peculiarity, the realistic channel capacity of present twisted subscriber line is calculated and the possibility that it is expanded and the ultra-limitation of capacity of HDSL are discussed.

It's discussed that the general receive \& deliver structure and some crucial technologies of HDSL, including CAP, Tomlinson Preceding, and DFE and so on, and the direction of subsequent work is indicated. The hardware circuits that are based on subscriber loop integrated service multiplex are also achieved. In addition, some problems about subscriber access network related to fiber channel are discussed.

There are some creative studies in this article:

Firstly, analytical method to calculate some parameters of symmetrical cable used as subscriber line is used. The analytical result in theory is consistent with actual result. This helps to analyze the effect of every factor in collective character.

Secondly, simple model to calculate pulse noises which affect subscriber channel be digitized is used. This provides data basis for the design of channel filter.

Thirdly, based on the theory of transmission line, the affection to subscriber channel be digitized when there is bridged tap in subscriber loop is analyzed.

Finally, the capacity in theory when average power is limited is estimated. Then noise and channel capacity in NEXT is gotten and the ultra-limitation of subscriber channel in theory in general condition is presented.

Let a casse-base $X=\left\{\mathrm{x}_{1}, \mathrm{~L}, \mathrm{x}_{m}, \mathrm{~L}, \mathrm{x}_{m}\right\}$ include $\mathrm{M}$ prior cases and a feature set $F=\left\{\mathrm{f}_{1}, \mathrm{~L}, \mathrm{f}_{m}, \mathrm{~L}, \mathrm{f}_{m}\right\}$ comprise $\mathrm{N}$ features in the problem description part of a case.A prior case $\mathrm{x}_{m}=\left(x_{m 1}, \mathrm{~L}, x_{m n}, \mathrm{~L}, x_{m N}\right)$ is composed of $\mathrm{N}$ feature values where $x_{m n}$ is the feature value of $f_{n}$ in $x_{m}$. Let $C=\left\{C_{1}, \mathrm{~L}, C_{i}, \mathrm{~L}, C_{c}\right\}$ be a set of the $\mathrm{C}$ clusters and $A=\left\{a_{1}, \mathrm{~L}, a_{i}, \mathrm{~L}, a_{c}\right\}$ be the set of the $\mathrm{C}$ cluster centers where $a_{i}=\left(a_{i 1}, \mathrm{~L}, a_{i n}, \mathrm{~L}, a_{i N}\right)$ is the cluster center of the itch cluster $C_{i}$ and $a_{i n}$ is the feature value of $f_{n}$ in $a_{i}$.Accordingly, the dissimilarity between a prior case $x_{m}$ and a cluster center $a_{i}$, termed as $\operatorname{diss}\left(x_{m}, a_{i}\right)$, can be defined as :

$$
\operatorname{diss}\left(x_{m}, a_{i}\right)=\sum_{n=1}^{N} w_{n} \times\left(x_{m n}-a_{i n}\right)^{2}=\sum_{n=1}^{N} w_{n} \times d\left(x_{m n}, a_{i n}\right)
$$

Where $w_{n} \in w$ is the feature weight of the feature $f_{n}$ and $w=\left\{w_{1}, \mathrm{~L}, w_{n}, \mathrm{~L}, w_{N}\right\}$ is the set of the $\mathrm{N}$ feature weights, $d\left(x_{m n}, a_{i n}\right)$ In addition, $d\left(x_{m n}, a_{i n}\right)$ is the difference between $x_{m}$ and $a_{i}$ in terms of feature $f_{n}$. The smaller the value of $\operatorname{diss}\left(x_{m}, a_{i}\right)$, the higher the probability that $x_{m}$ belongs to cluster $C_{i}$.

The objective of the WF-C-means algorithm, equivalent to $\mathrm{C}$-means, is to minimize the sum of the dissimilarities between all prior cases to their corresponding cluster centers, which can be expressed as follows:

$$
\left[\begin{array}{l}
y_{L}(z) \\
y_{H}(z)
\end{array}\right]=P(z)\left[\begin{array}{c}
x_{e}(z) \\
z^{-1} x_{0}(z)
\end{array}\right]
$$

subject to

$$
h(z)=h_{e}\left(z^{2}\right)+z^{-1} h_{0}\left(z^{2}\right)
$$

Where $\mathrm{U}$ is a matrix of size $M \times C$ that records the casecluster memberships and $u_{m i} \in\{1,0\}$ is an element in $\mathrm{U}$ that represents the membership of $x_{m}$ with the itch cluster $C_{i}$.If $u_{m i}=1, x_{m}$ belongs to $C_{i}$.If $u_{m i}=0$, by contrast, $x_{m}$ does not belong to $C_{i}$.The WF-C-means algorithm solves the described optimization problem by iteratively solving the following three reduced problems:

Problem $P_{1}:$ Fix $\mathrm{A}=\hat{A}$ and $\mathrm{w}=\hat{w}$ to solve the reduced problem $S(U, \hat{A}, \hat{w})$.

Problem $P_{2}$ : Fix $\mathrm{U}=\hat{U}$ and $\mathrm{w}=\hat{w}$ to solve the reduced problem $S(\hat{U}, A, \hat{w})$.

Problem $P_{3}:$ Fix $\mathrm{A}=\hat{A}$ and $\mathrm{U}=\hat{U}$ to solve the reduced problem $S(\hat{U}, \hat{A}, w)$.

The purpose of solving $P_{1}$ is to assign a prior case to a cluster whose cluster center is closest to the prior case, defined as Equation (4):

$$
\beta(z)=\left[\begin{array}{ll}
h_{e}(z) & g / o(z) \\
h_{0}(z) & g / 8(z)
\end{array}\right]
$$

Accordingly, the procedure for solving P2 is considered as a cluster-center updating procedure. Equation (5) can be used to calculate the solution of $\mathrm{P} 2$.

$$
a_{i n}=\sum_{m=1}^{M} u_{m i} \times x_{m n} / \sum_{m=1}^{M} u_{m i} \quad \text { for } 1 \leq \mathrm{i} \leq \mathrm{C}, 1 \leq \mathrm{n} \leq \mathrm{N}
$$

The difference between WF-C-Means and C-means is that WF-C-Means needs further solving the weight adjusting problem $P_{3}$ but C-means does not. The weight of a feature can be evaluated based on how the feature affects the quality of clustering result. In this research, the clustering quality is defined as the degree of minimizing the separations within clusters and maximizing the separations between clusters. Therefore, the problem $P_{3}$ is redefined as:

$$
\begin{array}{r}
\text { Maximize } \mathrm{V}(\hat{\mathrm{U}}, \hat{\mathrm{A}}, \mathrm{w}, \hat{\mathrm{g}})=\frac{\mathrm{S}^{\prime}(\hat{\mathrm{A}}, \mathrm{w}, \hat{\mathrm{g}})}{\mathrm{S}(\hat{\mathrm{U}}, \hat{\mathrm{A}}, \mathrm{w})} \\
=\frac{\sum_{n=1}^{N}\left[w_{n} \times\left(\sum_{i=1}^{C}\left\|C_{i}\right\| \times d\left(a_{i n}, g_{n}\right)\right)\right]}{\sum_{n=1}^{N}\left[w_{n} \times\left(\sum_{m=1}^{M} \sum_{i=1}^{C} u_{m i} \times d\left(x_{m n}, a_{i n}\right)\right)\right]}
\end{array}
$$

Subject to

$$
\left[\begin{array}{c}
x_{e}(z) \\
z^{-1} x_{0}(z)
\end{array}\right]=P(z)\left[\begin{array}{c}
y_{L}(z) \\
y_{H}(z)
\end{array}\right]
$$

Where $S(\hat{U}, \hat{A}, w)$ and $\mathrm{S}^{\prime}(\hat{\mathrm{A}}, \mathrm{w}, \mathrm{g})$ represent the sum of the 
separations within clusters and the sum of the separations between clusters, respectively. In addition, $g_{n}$ is the global center of all $\mathrm{M}$ prior cases in the case-base Expand $g_{n}$ is the feature value of $\mathrm{g}$ in terms of $f_{n}$ which can be obtained using $g_{n}=\sum_{m=1}^{M} x_{m n} / M$.In addition, $\left\|C_{i}\right\|$ represents the number of prior cases in the ith cluster $C_{i}$ such that $\sum_{i=1}^{C}\left\|C_{i}\right\|=M$

\section{LIFTING SCHEME}

The idea of lifting is based on the following: If a filter pair $(h, g)$ is complementary, then for every filter's the pair $(h, g)$, where

$$
\operatorname{Maximize} \mathrm{V}(\hat{\mathrm{U}}, \hat{\mathrm{A}}, \mathrm{w}, \hat{\mathrm{g}})=\frac{\sum_{\mathrm{n}=1}^{\mathrm{N}} \mathrm{w}_{\mathrm{n}} \times f_{n}}{\sum_{n=1}^{N} w_{n} \times e_{n}}
$$

is complementary, too. This rule also applies symmetrically as

$$
w_{n}^{\text {new }}=w_{n}+\Delta w_{n}=w_{n}+\frac{f_{n} / e_{n}}{\sum_{n=1}^{N}\left(f_{n} / e_{n}\right)} \quad \text { for } 1 \leq 1 \leq N
$$

The principle can be reversed, so that we can explicitly say: if the filter banks $(h, g)$ and $\left(h^{\prime}, g\right)$ allow for perfect reconstruction, then there exists a unique filter $s$ satisfying (8). Each such transform is called a lifting step- because what we perform is lifting the values of one particular subbed with the help of the other. In the language of polyphone matrices, lifting step described by (8) produces new polyphones matrix $\beta^{\text {kew }}(z)$, which is defined (based on (2) $-(5)$ as

$$
w_{n}^{\text {new }}=\frac{w_{n}+\frac{f_{n} / e_{n}}{\sum_{n=1}^{N}\left(f_{n} / e_{n}\right)}}{\sum_{n=1}^{N} w_{n}+\sum_{n=1}^{N}\left(\frac{f_{n}}{\sum_{n=1}^{N} f_{n}}\right)}
$$

Because we have lifted the low-pass subbed with the help of the high-pass subbed, this step is called primal lifting or update step. By taking (9) into consideration we can define

$$
\beta^{\text {rew }}(z)=\left[\begin{array}{cc}
1 & 0 \\
\ell /(0 z) & 1
\end{array}\right]
$$

By lifting the high-pass subbed with the help of the lowpass one, we have just made dual lifting or predict step (prediction of the odd samples from the even samples).

Lifting factorization is a generally defined process that is used to factorize complementary wavelet filter pair into a series of lifting steps. By computing greatest common divisor (gad) of even and odd filter values using the Euclidean algorithm, we obtain a structure consisting of subsequent pairs of primal and dual lifting steps

$$
P^{\prime}(z)=\prod_{i=1}^{m}\left[\begin{array}{cc}
1 & \xi_{i} /(z) \\
0 & 1
\end{array}\right]\left[\begin{array}{cc}
1 & 0 \\
\mathcal{P}_{i}(\phi z) & 1
\end{array}\right]\left[\begin{array}{cc}
K & 0 \\
0 & 1 / K
\end{array}\right]
$$

Where $\boldsymbol{s}_{i}(z)$ and $f_{i}(z)$ are Laurent polynomials computed for each step $\mathrm{i}$ by the gad algorithm as the resulting quotient (with the division remainder being zero). In practice, these are usually first or second order polynomials (one to three-tap FIR filters). $\mathrm{K}$ and $1 / \mathrm{K}$ act as resulting stream scaling constants and can be omitted if we accept the fact of resulting coefficients being scaled.

\section{LIFTING IMPLEMENTATION}

This assay is focuses on part of access network technology. The main contents are as the follows.

To discuss the function of the forthcoming copper loop, city cable channel's once and twice parameters are analyzed and calculated, and simple analytic model are used to describe the characteristics of channel. The transformation of parameter related to applied HDSL technology in higher band of frequency, and the influences of near reaction and twisted subscriber line are mainly analyzed.

To discuss the influence of transmission environment, the noise of the present subscribe channel and its influence on digital signal transmission are analyzed. The possible influence of pulse noise on HDSL technology's application is analyzed quantitatively, subscribe channel's interference voice is analyzed, and the influence of bridged tap on subscriber loop when it is applied under the digital condition of subscriber channel is calculated.

On the basis of above-mentioned analysis of channel peculiarity, the realistic channel capacity of present twisted subscriber line is calculated and the possibility that it is expanded and the ultra-limitation of capacity of HDSL are discussed.

There are some creative studies in this article:

Firstly, analytical method to calculate some parameters of symmetrical cable used as subscriber line is used. The analytical result in theory is consistent with actual result. This helps to analyze the effect of every factor in collective character.

Secondly, simple model to calculate pulse noises which affect subscriber channel be digitized is used. This provides data basis for the design of channel filter.

Thirdly, based on the theory of transmission line, the affection to subscriber channel be digitized when there is bridged tap in subscriber loop is analyzed.

Finally, the capacity in theory when average power is limited is estimated. Then noise and channel capacity in NEXT is gotten and the ultra-limitation of subscriber channel in theory in general condition is presented.

\section{EXPERIMENT AND DISCUSSION}

Integrated Fire/Flight/Propulsion Control System is one of the research emphases in the field of Aviation. Distributed simulation environment, as a design and experiment tool for study of IFFPCS, is needed urgently. Many factors have made the environment development more difficult, such as distributed sites of research members, complicated research 
content, heterogeneous hardware and software, and restricted related reference literature etc. To overcome these difficulties, an Integrated Control Distributed Simulation Environment is studied in this thesis.

Then, we proposed a hierarchical integration method for designing the longitudinal IFFPCS, with the purpose to reach minimum weapon aiming error. Based on relationships among components of propulsion system, model of propulsion system control integration is given. The model is integrated with flight control system. Their integrated model is integrated with fire control system again. Then fire control system, flight control system and propulsion control system, which are conventionally designed independently, are connected together through integrated fire/flight/propulsion coupler. Structure of IFFPCS is built, which lays a foundation for development of ICDSE.

Hierarchical distribution model of IFFPCS is put forward and structure of ICDSE which includes software structure and hardware structure is designed.

New concepts of distributed simulation computing and local real-time replay are presented specifically for overcoming the difficulty of remote distributed research members. Through frame synchronization mechanism during distributed simulation computing, all sub-modules in IFFPCS are set with same simulation step, and they must begin new calculation only after a frame synchronism signal is receive. In this way, executing paces of all sub-modules are regulated, and right causality among sub-modules under distributed environment is guaranteed. On the basis of distributed simulation computing, local real-time replay provides new ideas for dynamic debugging of simulation software and physical entity and study of distributed realtime simulation.

The fourth part describes the development history, current status and future layout of the Second Artillery Corps. It also analyzes network security problems there. Thence we draw a conclusion that the lack of network security insurance has become a bottleneck of restricting its network development. The fifth part puts forward a detailed network reinforcement project on the aspects of fire wall; anti-virus and invasion detect, and further emphasizes how to implement IDS based on network. We also discuss the necessity, urgency and feasibility of its network reinforcement. Middleware is state-of-the-art technique of distributed computing. Main Integrated Control Distributed Simulation Middleware's such as communication middleware, message misesteem manage middleware, and integration middleware are put forward and analyzed.

\section{CONCLUSIONS}

To tell in concrete terms, the dissertation discussed the drawbacks in the past reliability indices and system design, proposed several basic criteria on establishing the system reliability indices. Five indices and corresponding algorithms to analyze and bound the system reliability were posed in details.

Algorithms for computing the values or the bounds of the indices of the system availability and maintainability under path-criterion were put forward in order to get the indices of system perform ability, a theoretical model was established, equations and algorithms for solutions were deduced for computing the performances of a link with K-priorities traffics. Alterative algorithms were also given to analyze the performances of customers' telegraphic of three priorities. By means of the algorithms and formulae mentioned above, the values or the bounds of all the system reliability indices can be reckoned.

\section{ACKNOWLEDGMENT}

This work is supported by Scientific Research Fund of Heilongjiang Provincial Education Department (NO: 12531571).

\section{REFERENCES}

[1] Nick, J., Tuecke, S.: Sport and Economic Regeneration in Cities. Urban Studies, pp. $985--999$ (2005)

[2] Foster, I., Kesselman, C.: Old Locals and New Spaces. Leisure Studies, vol.24, pp.415--434 (2005)

[3] Lin, X.: Research on the Construction and Post-Games Utilization of Modern Olympic Venues. Journal of Beijing Sport University, vol.28, pp.1441--1444 (2005)

[4] Luo, P.: Research of the Management Profit of Large Sports Entertainment Yards in China. Journal of Xian University of Physical Education, vol.24, pp.17--20 (2007)

[5] Steinke, T.: Olympic Games Host City Marketing: An Exploration of Expectations and Outcomes. Sport Marketing Quarterly, Vol. 12, pp.37--47 (2003)

[6] Henrik Artman, Robert Ramberg, Hillevi Sundholm. A Case Study on Collaborative Design Learning [C].Computer Supported Collaborative Learing,2005 : 603-638.

[7] Xiang Chen, Min Li, Shuming Gao. A Web Services Based Platform for Exchange of Procedural CAD Models[C].The 9th International Conference on CSCW in Design,2005 : 605-610.

[8] Bidarra R, Neels W J, Bronsvoort W F. Boundary Evaluation for a Cellular Model[C]. Computers and Information in Engineering Conference, Chicago, USA, 2003 ; (1) : 255-265.

[9] Bidarra R, Bronsvoort W F. Semantic Feature Modeling[J]. Computer Aided Design, 2000 ; 32(3) : 201-225.

[10] Bronsvoort W F, Bidarra R, Nyirenda P J. Developments in Feature Modeling[J]. Computer-Aided Design and Application, 2006, 3(5): 655-664.

[11] Sun Lijuan and Liu Xianguo. Research on Algorithm of Computing Parameter Interval in Geometric Models[J]. Information Technology Journal, 2011, 10(2): 402-408.

[12] Den Berg E V, Bronsvoort W F. Validity Maintenance for Freeform Feature Modeling $[\mathrm{J}]$. Journal of Computing and Information Science in Engineering, 2010, 10(1): 1-14. 